\title{
Dynamical Analysis to Explain the Numerical Anomalies in the Family of Ermakov-Kalitkin Type Methods
}

\author{
Alicia Cordero ${ }^{a}$, Juan R. Torregrosa ${ }^{a}$ and Pura Vindel ${ }^{b}$ \\ ${ }^{a}$ Instituto de Matemática Multidisciplinar, Universitat Politècnica de València \\ Cno. de Vera s/n, 46022 Valencia, Spain \\ ${ }^{b}$ Instituto de Matemáticas y Aplicaciones de Castellón, Universitat Jaume I \\ Avenida de Vicent Sos Baynat s/n, 12071 Castellón de la Plana, Spain \\ E-mail(corresp.): acordero@mat.upv.es \\ E-mail: jrtorre@mat.upv.es \\ E-mail: vindel@uji.es
}

Received September 7, 2018; revised March 11, 2019; accepted March 12, 2019

\begin{abstract}
In this paper, we study the dynamics of an iterative method based on the Ermakov-Kalitkin class of iterative schemes for solving nonlinear equations. As it was proven in "A new family of iterative methods widening areas of convergence, Appl. Math. Comput.", this family has the property of getting good estimations of the solution when Newton's method fails. Moreover, the set of converging starting points for several non-polynomial test functions was plotted and they showed to be wider in the case of proposed methods than in Newton's case, for small values of the parameter. Now, we make a complex dynamical analysis of this parametric class in order to justify the stability properties of this family.
\end{abstract}

Keywords: nonlinear problems, iterative methods, complex dynamics, dynamical and parameter planes, critical points.

AMS Subject Classification: 65H05; 65H10; 37F10.

\section{Introduction}

Most of the problems faced by scientists and engineers involve equations that do not have a known analytical solution. Numerical methods are a good option to tackle and solve real world problems. In particular, iterative methods are used to find approximations of the solutions of the nonlinear equation $f(z)=0$, where $f: I \subseteq \mathbb{R} \rightarrow \mathbb{R}$ is defined in an open interval $I$. The best-known 
root-finding algorithm is Newton's method, which has order of convergence 2. However, many times Newton's method does not converge, or converges very slowly, to the roots of the equation. In [10], V.V. Ermakov and N.N. Kalitkin proposed a modification of Newton's scheme, checking that its behavior was better than Newton's method to find the solutions of certain nonlinear equations. The iterative expression is given by

$$
x_{k+1}=x_{k}-\beta_{k} \frac{f\left(x_{k}\right)}{f^{\prime}\left(x_{k}\right)}, \quad k=0,1, \ldots,
$$

where $\beta_{k}=\left\|f\left(x_{k}\right)\right\|^{2} /\left(\left\|f\left(x_{k}\right)\right\|^{2}+\left\|f\left(x_{k}-f\left(x_{k}\right) / f^{\prime}\left(x_{k}\right)\right)\right\|^{2}\right)$.

Following this scheme, in [5] a family of Ermakov-Kalitkin type of iterative methods for solving nonlinear problems was described. The iterative expression corresponding to this family, called PM family, is

$$
\begin{aligned}
& y_{k}=x_{k}-\alpha \frac{f\left(x_{k}\right)}{f^{\prime}\left(x_{k}\right)}, \\
& x_{k+1}=x_{k}-\frac{f\left(x_{k}\right)^{2}}{b f\left(x_{k}\right)^{2}+c f\left(y_{k}\right)^{2}} \frac{f\left(x_{k}\right)}{f^{\prime}\left(x_{k}\right)},
\end{aligned}
$$

where $b=\frac{1-\alpha+2 \alpha^{2}}{2 \alpha^{2}}$ and $c=\frac{1}{2 \alpha^{2}(\alpha-1)}$, where $\alpha \neq 0$ and $\alpha \neq 1$. The dumping factor at the second step, $f\left(x_{k}\right)^{2} /\left(b f\left(x_{k}\right)^{2}+c f\left(y_{k}\right)^{2}\right)$, is called in the following Kalitkin-type factor. The authors show that this family is third-order convergent and that, for specific nonlinear functions, its numerical behavior is better than Newton's method in certain situations. Furthermore, as it observed in [5], the performance of this family is better than that of Newton's method for systems of nonlinear equations in terms of the set of initial estimations converging to the solution.

Another way to analyze the behavior of a numerical method is to study it from a dynamical point of view, i.e., to consider the iterative method as a discrete dynamical system and to study its stability. This is a line of work that has proven to be especially fruitful in recent years (see, for example, the papers [1], [2], [6], [9], [11] and, more recently, [8], [12], [13] and [14]).

To carry out this dynamical study, the root-finding algorithm is applied on a low-degree polynomial $f$. By doing so, we obtain a rational map $R: \widehat{\mathbb{C}} \rightarrow \widehat{\mathbb{C}}$, where $\widehat{\mathbb{C}}$ denotes the Riemann sphere, whose dynamics describes how behaves the method when it is applied on function $f$. Indeed, the starting points which converge to the roots of $f$ by applying the numerical method are exactly those which converge to the roots of $f$ by iterating operator $R$.

Let us recall some definitions used in complex dynamics that will be useful in this work. A more complete study can be found in [3] and [15], for example.

Given a rational map $R: \widehat{\mathbb{C}} \rightarrow \widehat{\mathbb{C}}$, where $\widehat{\mathbb{C}}$ denotes the Riemann sphere, the set of its iterates can be considered as a discrete dynamical system. The orbit $\mathcal{O}(w)$ of a point $w \in \widehat{\mathbb{C}}$ is given by the subsequent iterates of $w$ under $R$, i.e.

$$
\mathcal{O}(w)=\left\{w, R(w), R^{2}(w), \ldots, R^{n}(w), \ldots\right\} .
$$

A point $z_{0} \in \widehat{\mathbb{C}}$ is called fixed if $R\left(z_{0}\right)=z_{0}$. A point $z_{0}$ is called periodic of period $p>1$ if $R^{p}\left(z_{0}\right)=z_{0}$ and $R^{k}\left(z_{0}\right) \neq z_{0}, k<p$. A point $z_{0}$ is pre-periodic 
if it is not periodic but it is eventually mapped under iteration of $R(z)$ to a periodic one.

Fixed points are classified depending on their multiplier $\lambda=R^{\prime}\left(z_{0}\right)$. A fixed point $z_{0}$ is called:

- attractor if $|\lambda|<1$ and superattractor if $\lambda=0$;

- repulsor if $|\lambda|>1$;

- indifferent or neutral if $|\lambda|=1$.

The multiplier of an indifferent fixed point $z_{0}$ can be written as $\lambda=R^{\prime}\left(z_{0}\right)=$ $e^{2 \pi i \theta}$ with $\theta \in[0,1)$. Depending on this $\theta$, an indifferent fixed point is called rationally indifferent (or parabolic) if $\theta=p / q$, where $p, q \in \mathbb{N}$ are coprime numbers; irrationally indifferent if $\theta \in \mathbb{R} \backslash \mathbb{Q}$.

If $R$ is obtained from a class of iterative methods depending on a parameter $\alpha$, the value of $\lambda$ also depends on $\alpha$. Therefore, we call $|\lambda|=\left|R^{\prime}\left(z_{0}, \alpha\right)\right|$ the stability function of the point $z_{0}$.

The same classification can be used for periodic points of any given period $p$ since they are fixed points of the map $R^{p}(z)$. The multiplier $\lambda$ of a fixed point $z_{0}$ determines the possible dynamics which might take place in a small neighborhood of it (see [15]). Those fixed points of rational function $R$ that are not roots of the polynomial $f$ are called strange fixed points.

The basin of attraction $\mathcal{A}\left(z_{0}\right)$ of an attracting point $z_{0}$ consists of the set of points $z \in \widehat{\mathbb{C}}$ that accumulate on $z_{0}$ under iteration of $R(z)$, i.e.

$$
\mathcal{A}\left(z_{0}\right)=\left\{z \in \widehat{\mathbb{C}}: R^{n}(z) \rightarrow z_{0} \text { when } n \rightarrow \infty\right\} .
$$

Similarly, the basin of attraction $\mathcal{A}\left(z_{0}\right)$ of a rationally indifferent point $z_{0}$ consists of the set of points $z \in \widehat{\mathbb{C}}$ that accumulate on $z_{0}$ under iteration of $R(z)$ but do not fall under iteration on $z_{0}$, i.e.

$$
\mathcal{A}\left(z_{0}\right)=\left\{z \in \widehat{\mathbb{C}}: R^{n}(z) \neq z_{0} \quad \forall n \in \mathbb{N}, R^{n}(z) \rightarrow z_{0} \text { when } n \rightarrow \infty\right\} .
$$

The dynamics of $R(z)$ provides a totally invariant partition of the Riemann sphere. The Fatou set, $\mathcal{F}(R)$, of a rational map $R(z)$ consists of the points $z \in \widehat{\mathbb{C}}$ such that the family of iterates of $R(z),\left\{R(z), R^{2}(z), \ldots, R^{n}(z), \ldots\right\}$, is normal in some open neighborhood $U$ of $z$. Its complement, the Julia set $\mathcal{J}(R)$, consists of the points where the dynamics of $R(z)$ is chaotic. The Fatou set is open and the Julia set is closed. The connected components of the Fatou set are called Fatou components and are mapped among themselves under iteration. Let us notice that, while the attractive fixed points are in the Fatou set, the indifferent fixed points are in Julia's set; therefore, although both have attractive basins, the pre-images of the indifferent fixed points are not in their basins of attraction, they must be in Julia's set. On the other hand, the number of basins of attraction of an indifferent fixed point depends on their multiplicity (the Leau-Fatou flower Theorem, see [15]). The union of the Fatou and Julia sets gives the dynamical plane of the rational function $R$.

The critical points of a rational map $R(z)$ are defined as the $z \in \widehat{\mathbb{C}}$ where $R(z)$ fails to be injective in any neighborhood of $z$ or, equivalently, the $z \in \widehat{\mathbb{C}}$ 
such that $R^{\prime}(z)=0$ (see [3], for example). Those critical points that do not correspond with any root of polynomial $f$ are called free critical points. All periodic Fatou components are related to critical points being that the basins of attraction of attracting and rationally indifferent points contain, at least, a critical point (see, for example, [15]). Moreover, when $R$ is obtained from a family of iterative methods depending on a parameter $\alpha$, a free critical point depending on $\alpha$ can be used as an initial estimation of $R$. Each value of $\alpha$ is related with a member of the class of iterative methods. In this case, a parameter plane is obtained by iterating a free critical point on $R$. In this parameter plane, we use different colors depending if the orbit of this critical point converges to the roots or not. So, when the orbit does not converge to the root, it means that there are some values of the parameter for which the corresponding numerical methods have pathological behavior.

The simplest example of complex dynamics is given by the dynamical system that appears by iterating Newton's method on polynomials of degree two. In this case, the Fatou set consists of two basins of attraction of the superattracting fixed points, corresponding to the roots of the polynomial, while the Julia set consists of a straight line which separates these basins of attraction. Nevertheless, this behavior becomes more complicated in the case of other families of iterative methods.

To better study the effectiveness of the proposed family of methods, in [5] the authors applied different and well-known methods to find the solutions of various nonlinear equations. Thereby, for example, in Figure 1 we see the dynamical planes of $f(x)=\arctan (x)-\frac{2 x}{1+x^{2}}$ for the different methods; the basins of attraction of the three solutions of the equation: $\xi_{1}=0, \xi_{2} \approx-1.3917$ and $\xi_{3} \approx 1.3917$ appear in blue, orange and green, respectively. For Newton's scheme (see Figure 1 (a)), it was observed that these areas of convergence were bounded in the plotted region, $[-6,6] \times[-6,6]$.

The small black regions in Newton's dynamical plane did not correspond to strange fixed points, but to pre-images of the infinity. When Traub's method was used (see Figure 1 (c)), the black basin (convergence to the infinity) was "crossed" by orange regions of convergence to zero. Moreover, the pre-images of the infinity inside the bounded region of convergence were bigger than in case of Newton's. In Kalitkin's dynamical plane (see Figure 1 (b)), the real initial estimations with convergence to the roots were able to be very far from them, but with a complicated dynamics. The dynamical plane associated to Ostrowski's procedure was clean and the basins were wider than in previous cases. Indeed, there were no convergence to the infinity in the area under analysis.

These figures are obtained by using the routines described in [7]. To draw these images, a mesh of $400 \times 400$ initial points has been used, 80 was the maximum number of iterations involved and $10^{-3}$ the tolerance used as the stopping criterion. In this paper, we have used a white star to show the roots of the nonlinear system. A color is assigned to each initial estimation (each point of the mesh) depending on where they converge to, brighter as lower is the number of iterations needed to converge, and black color is adopted when the maximum number of iterations is reached or the process diverges. 


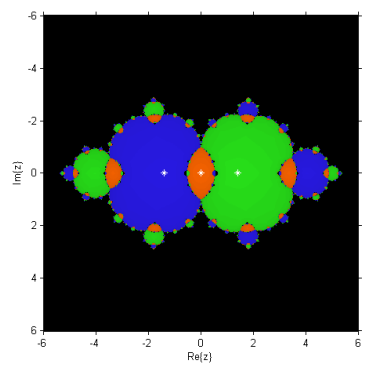

(a) Newton

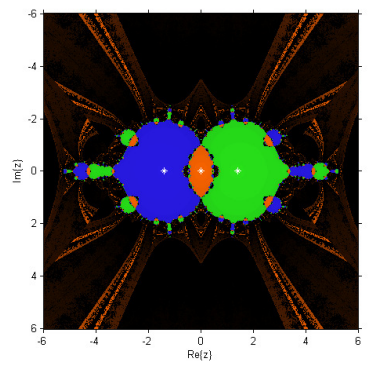

(c) Traub

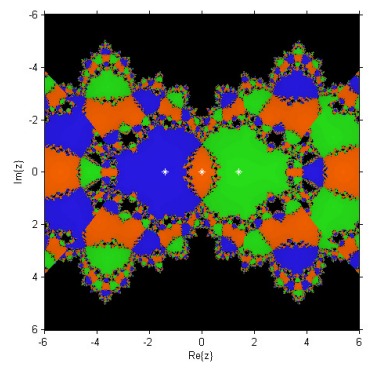

(b) Kalitkin

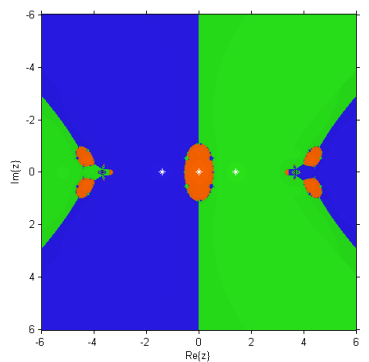

(d) Ostrowski

Figure 1. Dynamical planes of $f(x)=\arctan (x)-\frac{2 x}{1+x^{2}}$

Let us recall that the iterative scheme of the second-order Newton's method is

$$
x_{k+1}=x_{k}-\frac{f\left(x_{k}\right)}{f^{\prime}\left(x_{k}\right)}, \quad k=0,1, \ldots
$$

The iterative expression of Traub's method (see [17]), which has order of convergence three, is

$$
\begin{aligned}
& y_{k}=x_{k}-f\left(x_{k}\right) / f^{\prime}\left(x_{k}\right), \\
& x_{k+1}=x_{k}-\left(f\left(x_{k}\right)+f\left(y_{k}\right)\right) / f^{\prime}\left(x_{k}\right), \quad k=0,1, \ldots,
\end{aligned}
$$

and Ostrowski's method ( [16]), whose order of convergence is four, is

$$
\begin{aligned}
& y_{k}=x_{k}-f\left(x_{k}\right) / f^{\prime}\left(x_{k}\right), \\
& x_{k+1}=y_{k}-\frac{f\left(x_{k}\right)}{f\left(x_{k}\right)-2 f\left(y_{k}\right)} \frac{f\left(y_{k}\right)}{f^{\prime}\left(x_{k}\right)}, \quad k=0,1, \ldots
\end{aligned}
$$

When the performance of the elements of PM family (1.1) was observed on $f(x)=\arctan (x)-\frac{2 x}{1+x^{2}}([5])$, the stability of the methods seemed to be directly related with the absolute value of parameter $\alpha$. In fact, the best results were obtained for $\alpha=-0.15$ (Figure $2(\mathrm{c})$ ) and $\alpha=0.15$ (Figure 2 (d)), being the first of these cases much more stable than classical methods as Newton, Traub or even Ostrowski. This behavior was also found in [5] for 
other nonlinear functions, being always the best elements of the family those with $\alpha$ close to zero.

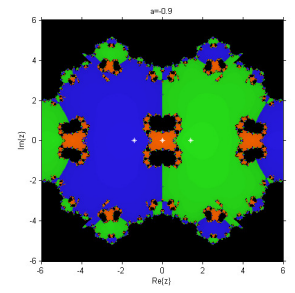

(a) $\alpha=-0.9$

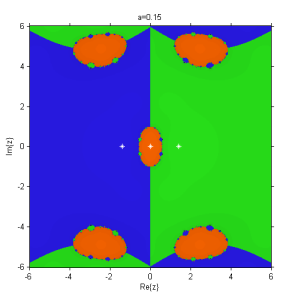

(d) $\alpha=0.15$

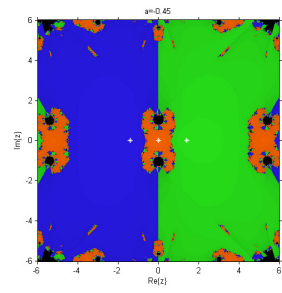

(b) $\alpha=-0.45$

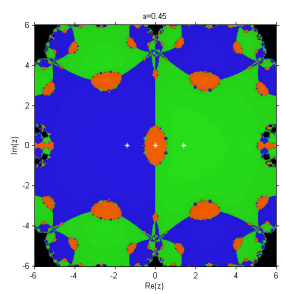

(e) $\alpha=0.45$

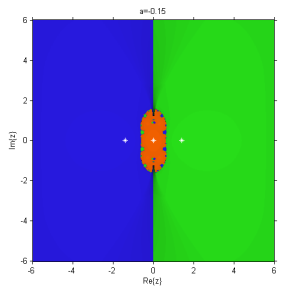

(c) $\alpha=-0.15$

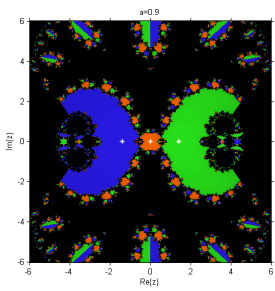

(f) $\alpha=0.9$

Figure 2. Dynamical planes of some members of PM class on $f(x)=\arctan (x)-\frac{2 x}{1+x^{2}}$

As these tests show, PM family has the property of getting good estimations of the solution when Newton's method fails. This behavior is not completely understood from a numerical point of view as it is only tested on some specific functions and the conclusions are not generalizable. Now, we make a deep dynamical analysis with the aim of understanding the stability properties of this family that justify these features.

Thus, we start the dynamical study of this class by calculating its fixed and critical points in Section 2; in Section 3, we explain the parameter planes which represent this family and describe the dynamical planes associated to certain values of the parameter. This last description allows us to explain the reasons for the stability of the family for small values of the parameter, in Section 3.1. Finally, we state some conclusions.

\section{Fixed and critical points}

We are going to present a dynamical study of PM family, designed in [5] whose expression is given in (1.1), applied on quadratic polynomial $p(z)=(z-a)(z-$ $b)$. The fixed point function associated to PM family on $p(z)$ is

$$
\begin{aligned}
& g(z, \alpha, a, b)=z+\frac{2 \alpha^{2}(z-a)^{3}(z-b)^{3}}{(a+b-2 z)(a-z)^{2}(b-z)^{2} M(z, \alpha, a, b)}, \\
& M(z, \alpha, a, b)=(\alpha-1)\left(1-\alpha+2 \alpha^{2}\right)(a+b-2 z)^{4}+(a(1-\alpha) \\
& +b+(-2+\alpha) z)^{2}(a+b(1-\alpha)+(-2+\alpha) z)^{2} .
\end{aligned}
$$


It is known (see, for example, [3,4]) that the roots of a polynomial can be transformed by a map $h(z)=\frac{z-a}{z-b}$ with no qualitative changes on the dynamics of the family of polynomials. By applying this conjugacy map

$$
G=h \circ g \circ h^{-1},
$$

the operator of the PM class is conjugated to the rational function

$$
G(z, \alpha)=z^{3} \frac{\alpha^{2}+2(1+z)^{2}(-1+\alpha)}{\alpha^{2} z^{2}+2(1+z)^{2}(-1+\alpha)} .
$$

As we have pointed before, this family has the property of getting good estimations of the solution when Newton's method fails. Our dynamical analysis deeps in the understanding of the stability properties of this family that justify this behavior. The first step of this dynamical study of operator $G(z, \alpha)$, as a function of parameter $\alpha$, consists of calculating its fixed and critical points. As we will see, the number and the stability of the fixed and critical points depend on parameter $\alpha$. It is known that any rational map of degree $d$ has $d+1$ fixed points (including multiplicity) and $2 d-2$ critical points (with multiplicity) (see [3], for example). In our case, this results in 6 fixed and 8 critical points.

As fixed points satisfy

$$
G(z, \alpha)=z,
$$

it can be easily calculated that the fixed points of $G(z, \alpha)$ are $z=0, z=\infty$ (both corresponding to the original roots of polynomial $p(z)$ ) and also $z=1$ and $z=-1$, with multiplicity 3 . These last two points are called strange fixed points.

$$
G(z, \alpha)=z \quad \Leftrightarrow \quad 2 z(\alpha-1)(z-1)(z+1)^{3}=0 .
$$

To study the stability of these fixed points, we need the derivative of operator $G(z, \alpha)$,

$$
G^{\prime}(z, \alpha)=z^{2} \frac{\alpha^{4} z^{2}+12(1+z)^{4}(1-2 \alpha)+2 \alpha^{2}(1+z)^{2} Q(z, \alpha)}{\left(\alpha^{2} z^{2}+2(1+z)^{2}(-1+\alpha)\right)^{2}},
$$

where $Q(z, \alpha)=\alpha\left(3-2 z+3 z^{2}\right)+\left(3+14 z+3 z^{2}\right)$.

Proposition 1. Given $\alpha=a+i b, a, b \in \mathbb{R}$, the stability of fixed point $z=1$ satisfies the following statements:

1. $z=1$ is an attractor, that is $\left|G^{\prime}(1, \alpha)\right|<1$ if any of the following conditions is satisfied: (i) $a \leq-17$, (ii) $-17<a<4(-2-\sqrt{5})$ and $-\sqrt{\frac{-16+32 a-15 a^{2}-a^{3}}{17+a}}<b<\sqrt{\frac{-16+32 a-15 a^{2}-a^{3}}{17+a}}$, (iii) $4(-2+\sqrt{5})<a<$ 1 and $-\sqrt{\frac{-16+32 a-15 a^{2}-a^{3}}{17+a}}<b<\sqrt{\frac{-16+32 a-15 a^{2}-a^{3}}{17+a}}$.

2. $z=1$ is indifferent, that is $\left|G^{\prime}(1, \alpha)\right|=1$, if

$$
16+15 a^{2}+a^{3}+17 b^{2}+a\left(-32+b^{2}\right)=0 .
$$

3. $z=1$ is a repulsive fixed point in any other case. 
Proof. From the expression of the derivative of the operator, it can be deduced that:

$$
G^{\prime}(1, \alpha)=\frac{\alpha^{2}+24 \alpha-24}{\alpha^{2}+8 \alpha-8} .
$$

So, given $\alpha=a+i b, a, b \in R$, we study when $\left|G^{\prime}(1, \alpha)\right|=1$; so, $z=1$ is an indifferent fixed point if

$$
16+15 a^{2}+a^{3}+17 b^{2}+a\left(-32+b^{2}\right)=0 .
$$

This curve corresponds to the values of parameter $\alpha$ where the fixed point $z=1$ is an indifferent point and this curve can be separated in two different parts, that can be observed in Figure 3.

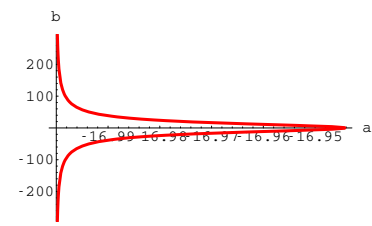

(a) Real negative values

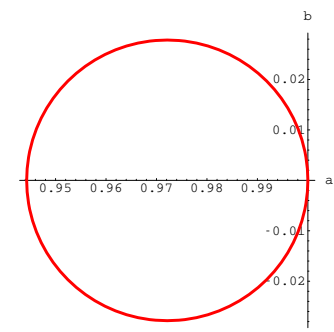

(b) Real positive values

Figure 3. Loci of $z=1$ being an indifferent fixed point

Condition ( $\mathrm{ii}$ ) for the attracting behavior of $z=1$ is represented in Figure 3(a), meanwhile condition (iii) is observed in Figure 3(b). It can be easily checked that $z=1$ is an attractor if $\alpha=a+i b$ satisfies one of the conditions (i), $(i i)$ or $(i i i)$. Otherwise, it is a repulsive fixed point.

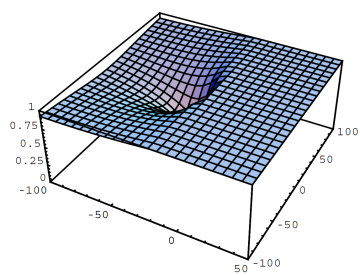

(a) Real negative values of $\alpha$

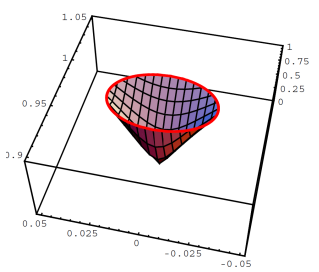

(b) Real positive values of $\alpha$

Figure 4. Stability function for $z=1$

The stability function for $z=1,\left|G^{\prime}(1, \alpha)\right|$, can also be observed in Figure 4 for positive and negative values of parameter $\alpha$. Moreover, it can be observed that $\left|G^{\prime}(1, \alpha)\right|=1$ for values of $\alpha$ on the curves of Figure 3. In it, the complex values of parameter $\alpha$ where the strange fixed point $z=1$ is attracting are observed; those values must be avoided in the practical use of the members of the class of iterative methods, as they lead to divergent behavior.

In a similar way, the following result can be proven. 
Proposition 2. Fixed point $z=-1$ is an indifferent point of $G(z, \alpha)$ for every value of the parameter $\alpha \in \hat{\mathbb{C}}$. In addition, it is always on the boundary of two basins of attraction.

Proof. It is easy to check that $\left|G^{\prime}(-1, \alpha)\right|=1$. On the other hand, from expression (2.1) we see that the multiplicity of $z=-1$ is three, the Leau-Fatou flower Theorem (see [15]) assures that $z=-1$ is in the intersection of two disjoint attractive basins.

From equation (2.2), we obtain that the number of different critical points of rational operator $G(z, \alpha)$ is eight, that correspond to $z=0$ and $z=\infty$ (both of them with multiplicity 2) and also four free critical points, whose analytical expressions are

$$
\begin{aligned}
& c_{1}(\alpha)=0.5\left(C_{1}(\alpha)+\sqrt{C_{1}^{2}(\alpha)-4}\right)=1 / c_{2}(\alpha), \\
& c_{3}(\alpha)=0.5\left(C_{2}(\alpha)+\sqrt{C_{2}^{2}(\alpha)-4}\right)=1 / c_{4}(\alpha),
\end{aligned}
$$

where

$$
\begin{aligned}
& C_{1}(\alpha)=\frac{-4(-1+\alpha)\left(-6+6 \alpha+\alpha^{2}\right)-\alpha^{2} \sqrt{2(-1+\alpha)\left(26-26 \alpha+3 \alpha^{2}\right)}}{6(-1+\alpha)\left(-2+2 \alpha+\alpha^{2}\right)}, \\
& C_{2}(\alpha)=\frac{-4(-1+\alpha)\left(-6+6 \alpha+\alpha^{2}\right)+\alpha^{2} \sqrt{2(-1+\alpha)\left(26-26 \alpha+3 \alpha^{2}\right)}}{6(-1+\alpha)\left(-2+2 \alpha+\alpha^{2}\right)} .
\end{aligned}
$$

Proposition 3. The number of critical points decreases only for some values of the parameter:

$$
\alpha \in\left\{-1 \pm \sqrt{3}, 2(-2 \pm \sqrt{6}), 2(-6 \pm \sqrt{42}), \frac{13}{3} \pm \frac{1}{3} \sqrt{91}\right\},
$$

where they coincide two-by-two.

Proof. The degree of a rational function decreases if the roots of the numerator and denominator coincide or if the coefficient of the higher degree term vanishes. From expression (2.2), the highest-degree term of $G^{\prime}(z, \alpha)$ is

$$
6(\alpha-1)\left(2 \alpha+\alpha^{2}-2\right) z^{6}
$$

So, if $2 \alpha+\alpha^{2}-2=0$, that is, if $\alpha=-1 \pm \sqrt{3}$, the degree of the rational function $G^{\prime}(z, \alpha)$ is five. In fact, the expressions of the operator and its derivative for these value are

$$
G(z,-1 \pm \sqrt{3})=z^{4} \frac{2+z}{1+2 z}, \quad G^{\prime}(z,-1 \pm \sqrt{3})=z^{3} \frac{8 z^{2}+17 z+8}{(2 z+1)^{2}}
$$

and there are only two free critical points at $z=\frac{-17 \pm \sqrt{33}}{16}$.

On the other hand, if $-2+2 \alpha+\alpha^{2} \neq 0$, the number of different critical points decreases if one of the following items is satisfied: 
(a) $c_{1}(\alpha)=c_{2}(\alpha) \Leftrightarrow C_{1}^{2}(\alpha)=4$. From

$$
\frac{-4(-1+\alpha)\left(-6+6 \alpha+\alpha^{2}\right)-\alpha^{2} \sqrt{2(1-\alpha)\left(26-26 \alpha+3 \alpha^{2}\right)}}{6(-1+\alpha)\left(-2+2 \alpha+\alpha^{2}\right)}=2
$$

it is deduced

$$
-16(\alpha-1)\left(3 \alpha+\alpha^{2}-3\right)=\alpha^{2} \sqrt{2(1-\alpha)\left(26-26 \alpha+3 \alpha^{2}\right)} .
$$

By eliminating the square root and simplifying, it is obtained

$$
2^{7}(1-\alpha)\left(3 \alpha+\alpha^{2}-3\right)^{2}=\alpha^{4}\left(26-26 \alpha+3 \alpha^{2}\right) .
$$

Therefore,

$$
-3\left(2 \alpha+\alpha^{2}-2\right)\left(8 \alpha+\alpha^{2}-8\right)\left(24 \alpha+\alpha^{2}-24\right)=0
$$

and this is true only if

$$
\alpha=2(-2 \pm \sqrt{6}) \quad \text { or } \quad \alpha=2(-6 \pm \sqrt{42}) .
$$

(b) $c_{3}(\alpha)=c_{4}(\alpha) \Leftrightarrow C_{2}^{2}(\alpha)=4$.

The result is the same as previous one. So, $\alpha=2(-2 \pm \sqrt{6})$ or $\alpha=$ $2(-6 \pm \sqrt{42})$.

(c) $c_{1}(\alpha)=c_{3}(\alpha)$ and $c_{2}(\alpha)=c_{4}(\alpha) \Leftrightarrow C_{1}(\alpha)=C_{2}(\alpha)$.

In this case, $2(1-\alpha)\left(26-26 \alpha+3 \alpha^{2}\right)=0$, so the only values satisfying this equality are $\alpha=1$ and $\alpha=\frac{13}{3} \pm \frac{1}{3} \sqrt{91}$.

Remark 1. As we have proven, the number of critical points decreases for some values of parameter $\alpha$. At this point, some aspects of the resulting rational function must be pointed out:

(i) If $\alpha=-1 \pm \sqrt{3}$, the denominator of the critical points vanishes and it can be checked that one of them goes to $z=\infty$, another one goes to $z=0$ and only two free critical points remain.

(ii) When $\alpha=2(-6 \pm \sqrt{42})$ and $\alpha=2(-2 \pm \sqrt{6}), c_{1}(\alpha)=c_{2}(\alpha)=1$ and the fixed point $z=1$ becomes a superattractor.

(iii) If $\alpha=\frac{13}{3} \pm \frac{1}{3} \sqrt{91}$, then $c_{1}(\alpha)=c_{3}(\alpha)=\frac{-11+i \sqrt{455}}{24}$ and $c_{2}(\alpha)=c_{4}(\alpha)=$ $\frac{-11-i \sqrt{455}}{24}$ so the number of free critical points is two, being only one of them independent. 


\section{Parameter and dynamical planes}

As we have previously stated, the dynamical behavior of operator $G(z, \alpha)$ depends on the value of parameter $\alpha$ and it is deduced by following the orbit of a free critical point. In this family there are four critical points, but only two of them are independent as they are two-by-two conjugated. The fact that they are conjugated implies that if one critical orbit converges to $z=0$ then the other one converges to $z=\infty$; therefore, it is enough to analyze the asymptotic behavior of one of the conjugated critical orbits, i.e. the orbits of the free independent critical points, in order to study the existence of any attractor other than the roots, $z=0$ and $z=\infty$. In fact, we only have two different parameter planes, as can be seen in Figure 5 .

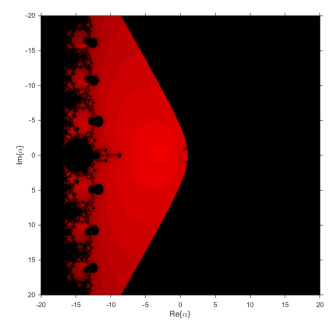

(a) $c_{1}(\alpha)$ or $c_{2}(\alpha)$ used as initial estimations

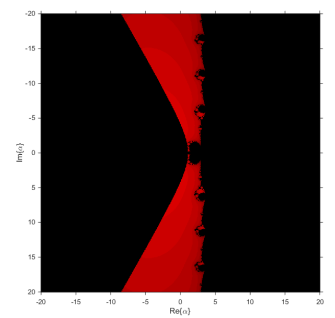

(c) $c_{3}(\alpha)$ or $c_{4}(\alpha)$ used as initial estimations

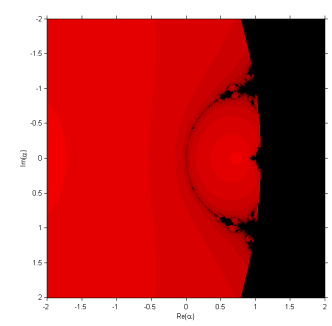

(b) A detail of (a)

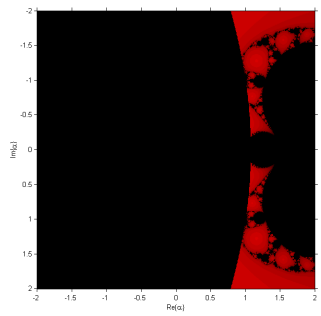

(d) A detail of (c)

Figure 5. Parameter planes of operator $G(z, \alpha)$

To generate these parameter planes, a mesh of $2000 \times 2000$ points is used. Each point of this mesh corresponds to a value of $\alpha$ and, therefore, to a member of the PM family. For each point of the mesh, the corresponding scheme uses, as initial estimation a free independent critical point. Red color is assigned to each point of the mesh if it converges to one of the roots (that is, 0 or $\infty$ ) and in black color in other case.

These planes show us the reason for the bad general behavior of this family, since for all $\alpha$ values there are at least two free critical points that will be in basins of attraction different from those of the roots. However, as we will see below, these "dangerous" regions of attraction will be very small for values of $\alpha$ close to zero.

Firstly, our aim is to study the dynamical planes for this family. Firstly, we study the singular cases where the number of critical points decrease. 


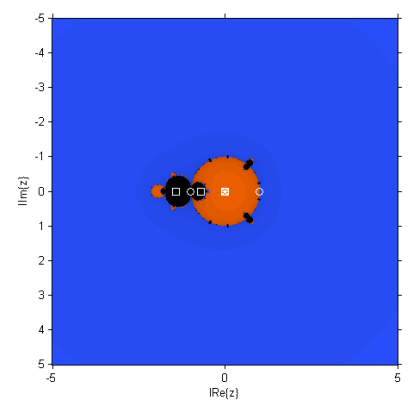

(a) $\alpha=-1 \pm \sqrt{3}$

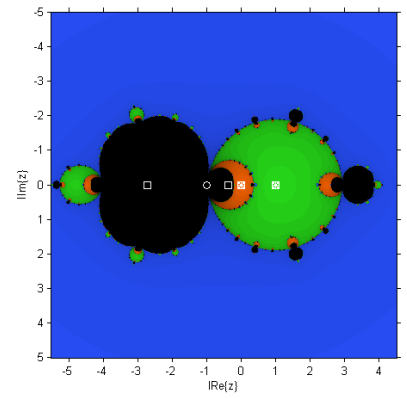

(c) $\alpha=2(-6 \pm \sqrt{42})$

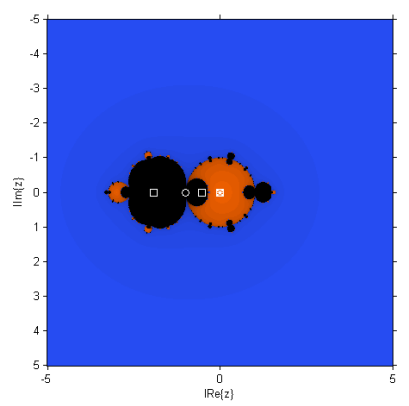

(b) $\alpha=2(-2 \pm \sqrt{6})$

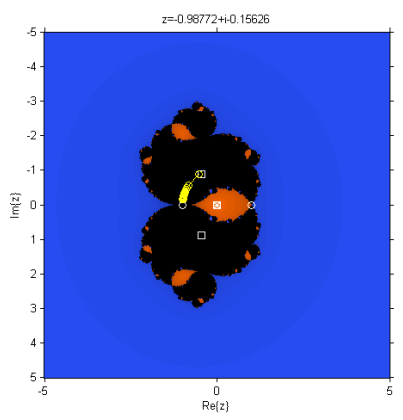

(d) $\alpha=\frac{13}{3} \pm \frac{1}{3} \sqrt{91}$

Figure 6. Dynamical planes of the operator $G(z, \alpha)$

As we have proven previously, operator $G(z, \alpha)$ is reduced for the following values of the parameter

$$
\alpha \in\left\{-1 \pm \sqrt{3}, 2(-2 \pm \sqrt{6}), 2(-6 \pm \sqrt{42}), \frac{13}{3} \pm \frac{1}{3} \sqrt{91}\right\} .
$$

Let us recall that we do not consider values $\alpha=0$ and $\alpha=1$, since PM family of iterative methods is not defined for them. The expressions of the operator for the four first values of the parameter are:

$$
\begin{aligned}
G(z,-1 \pm \sqrt{3}) & =z^{4} \frac{2+z}{1+2 z}, \\
G(z, 2(-2 \pm \sqrt{6})) & =-z^{3} \frac{3+z}{1+3 z},
\end{aligned}
$$

where we can verify that they are simpler or even of higher order of convergence (for quadratic polynomials), as is the case of $\alpha=-1 \pm \sqrt{3}$.

For these values of the parameter, there are only two free critical points, so, there exists at least one basin of attraction besides those of the roots; their dynamical planes can be seen in Figure 6 . In both, $z=1$ and $z=-1$ are indifferent points; however, only $z=-1$ has two basins of attraction because of it has multiplicity 3 , the black areas around $z=-1$ in Figure 6 (b). 
For $\alpha=2(-6 \pm \sqrt{42})$, two critical points collapse in $z=1$, and this fixed point becomes a superattractor (see Figure 6 (c)); when $\alpha=\frac{13}{3} \pm \frac{1}{3} \sqrt{91}$, there are two free critical points but with double multiplicity, which implies the existence of two large basins of attraction, in addition to those of 0 and $\infty$, its dynamical plane can be seen in Figure $6(\mathrm{~d})$. The corresponding operators are:

$$
\begin{aligned}
G(z, 2(-6 \pm \sqrt{42})) & =z^{3} \frac{-11+2 z+z^{2}}{1+2 z-11 z^{2}} \\
G\left(z, \frac{13}{3} \pm \frac{1}{3} \sqrt{91}\right) & =z^{3} \frac{16+6 z+3 z^{2}}{3+6 z+16 z^{2}}
\end{aligned}
$$

For any other value of the parameter, different from the previous ones, there are four free critical points; so, there are three or four attraction basins different from those of the roots. Two examples can be seen in Figure 7: in the first case, corresponding to $\alpha=3$, all the black area is the basin of attraction of $z=-1$, with four critical points inside (Figure 7 (a)) and $z=1$ has not basin of attraction as it lays in the Julia set; in Figure 7 (b), there are two kinds of black regions, those close to $z=-1$ corresponding to its basins of attraction and other that has $z=1$ in their boundary; the later ones form the basin of attraction of a periodic orbit of period 2 .

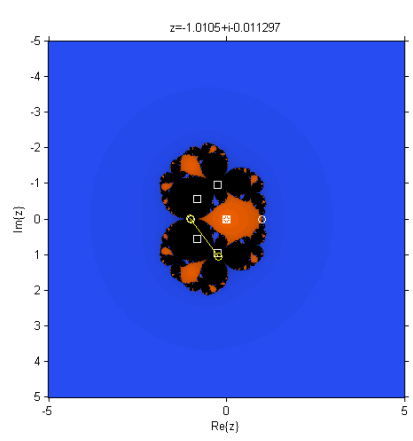

(a) $\alpha=3$

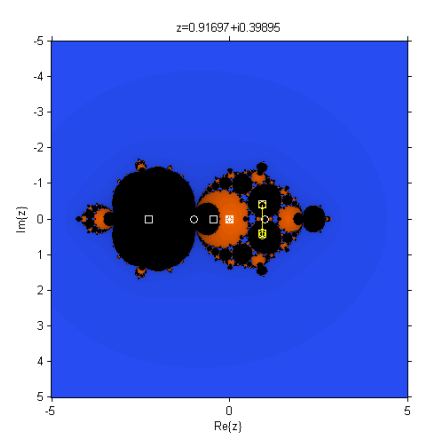

(b) $\alpha=-15$

Figure 7. Dynamical planes of the operator $G(z, \alpha)$ with four different free critical points

\subsection{Dynamical planes for small values of the parameter}

To conclude, we study the case of the rational function when $\alpha=0$ or it is very close to be null. Although in this case the family of iterative methods is not defined, it is useful for the sake of continuity of the rational function, to analyze this case. For $\alpha=0$ the operator is reduced to $G(z, 0)=z^{3}$ and so, its dynamical plane is divided in two parts by the unity circle. It is a good explanation of why this method works well for small absolute values of the parameter. Indeed, if we study the dynamical planes for small values of $\alpha$, it is observed that the basins of attraction that do not correspond to the roots of the polynomial are really small. For small values of the parameter $\alpha$ the dynamical plane can be seen in Figure 8 . 


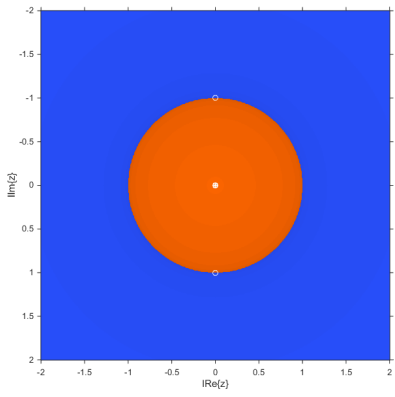

(a) $\alpha=0$

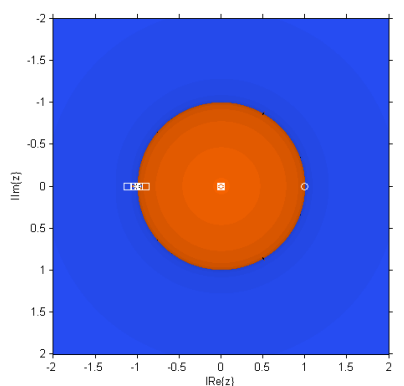

(b) $\alpha=-0.1$

Figure 8. Dynamical planes of operator $G(z, \alpha)$ for $\alpha \approx 0$

Nevertheless, if we see in detail the dynamical planes around $z=-1$, we observe that the region of non-convergence to the roots increases as the value of $\alpha$ gets higher in absolute value (see Figure 9). It is better understood, then, why this method has a good dynamical behavior when small values of the parameter are used: in this case the non-convergence region is very small and the good performance of the Kalitkin-type factor in the iterative expression still holds.

Finally, the following result can be concluded from the dynamical planes showed in Figure 9. As it is deduced from Figure 5, the critical points $c_{1}(\alpha)$ and $c_{2}(\alpha)$ are in the basins of attraction of $z=0$ and $z=\infty$, respectively. Moreover, $c_{3}(\alpha)$ and $c_{4}(\alpha)$ are in the basin of attraction of $z=-1$. This behavior is held until $\alpha$ reaches a bifurcation locus, which happens for values of $\alpha$ close to one.

Proposition 4. For small values of parameter $\alpha$, two free critical points are located in the two basins of attraction of the indifferent point $z=-1$ and the other two are in the basins of attraction of the roots.

This allows us to state that there is no other attraction region outside those two small basins of attraction that correspond to the fixed point rationally indifferent $z=-1$. Therefore, to ensure the convergence to the roots of the polynomial, it is sufficient to choose a value of $\alpha$ close to zero and, in case of quadratic polynomials, to avoid initial estimations close to $z=-1$, see Figure 9 .

\section{Conclusions}

We have analyzed the dynamical behavior of a class of iterative methods that has, for some problems, better numerical performance than Newton's method. Complex dynamical analysis has allowed us to understand the reason why it happens. The key fact is the existence of some free critical points in the boundary of a rational indifferent fixed point that share an only basin of attraction. This basin is, in general, the source of very unstable behavior but its wideness is extremely reduced when the value of $\alpha$ is almost null. 


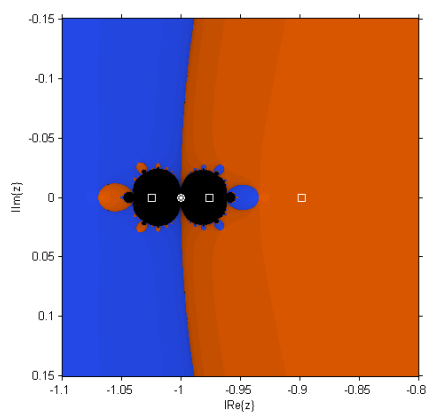

(a) $\alpha=0.1$

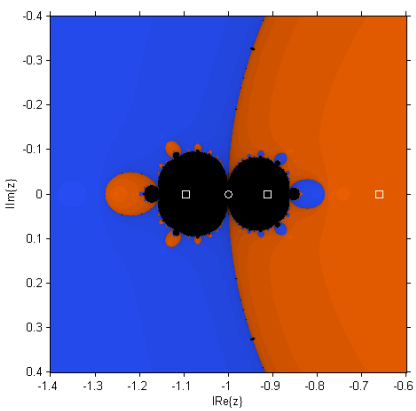

(c) $\alpha=0.3$

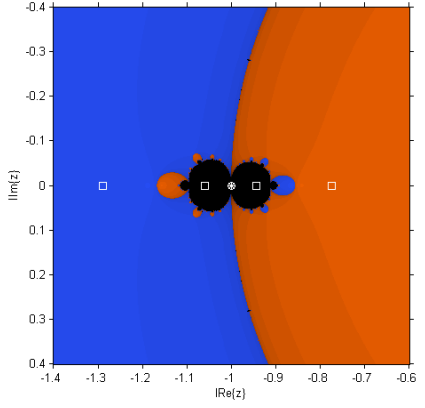

(b) $\alpha=0.2$

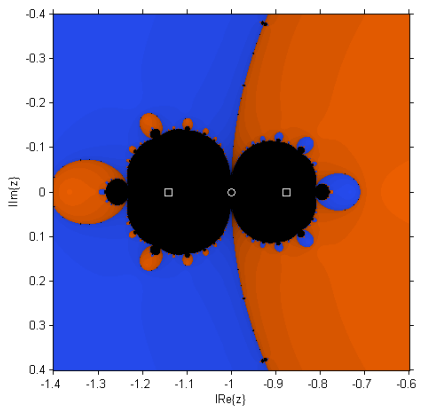

(d) $\alpha=0.4$

Figure 9. Zoom of different dynamical planes for small values of $\alpha$

\section{Acknowledgements}

This research was supported by Spanish Ministry grant MTM2014-52016-C022-P, Generalitat Valenciana PROMETEO/2016/089 and UJI project P1.1B20115 16. The authors also want to thank the anonymous referees for their suggestions and comments that have improved the final version of the paper.

\section{References}

[1] S. Amat, S. Busquier and S. Plaza. On the dynamics of a family of third-order iterative functions. ANZIAM, 48:343-359, 2007.

[2] I. Argyros and Á.A. Magreñán. A study on the local convergence and the dynamics of Chebyshev-Halley type methods free from second derivative. Numer. Algor., 71:1-23, 2016. https://doi.org/10.1007/s11075-015-9981-x.

[3] A.F. Beardon. Iteration of rational functions. Graduate Texts in Mathematics. Springer-Verlag, New York, 1991. https://doi.org/10.1007/978-1-4612-4422-6.

[4] P. Blanchard. Complex analytic dynamics on the Riemann sphere. Bull. AMS, 11(1):85-141, 1984. https://doi.org/10.1090/S0273-0979-1984-15240-6. 
[5] D.A. Budzko, A. Cordero and J.R. Torregrosa. A new family of iterative methods widening areas of convergence. Appl. Math. Comput., 252:405-417, 2015. https://doi.org/10.1016/j.amc.2014.12.028.

[6] B. Campos, A. Cordero, J.R. Torregrosa and P. Vindel. Dynamics of the family of c-iterative methods. Inter. J. Comput. Math., 92(9):1815-1825, 2015. https://doi.org/10.1080/00207160.2014.893608.

[7] F.I. Chicharro, A. Cordero and J.R. Torregrosa. Drawing dynamical and parameter planes of iterative families and methods. Sci. World J., 2013(ID 780153):11, 2013.

[8] C. Chun and B. Neta. Comparative study of methods of various orders for finding repeated roots of nonlinear equations. Comput. Appl. Math., 340:11-42, 2018. https://doi.org/10.1016/j.cam.2018.02.009.

[9] A. Cordero, J. García-Maimó, J.R. Torregrosa, M.P. Vassileva and P. Vindel. Chaos in King's iterative family. Appl. Math. Letters, 26:842-848, 2013. https://doi.org/10.1016/j.aml.2013.03.012.

[10] V.V. Ermakov and N.N. Kalitkin. The optimal step and regularization for Newton's method. USSR Computational Mathematics and Mathematical Physics, 21(2):235-242, 1981. https://doi.org/10.1016/0041-5553(81)90022-7.

[11] J.M. Gutiérrez, M.A. Hernández and N. Romero. Dynamics of a new family of iterative processes for quadratic polynomials. Comput. Appl. Math., 233:26882695, 2010. https://doi.org/10.1016/j.cam.2009.11.017.

[12] Y.I. Kim, R. Behl and S.S. Motsa. An optimal family of eighth-order iterative methods with an inverse interpolatory rational function error corrector for nonlinear equations. Math. Model. Anal., 22(3):321-336, 2017. https://doi.org/10.3846/13926292.2017.1309585.

[13] M-Y. Lee, Y.I. Kim and Á.A. Magreñán. On the dynamics of a triparametric family of optimal fourth-order multiple-zero finders with a weight function of the principal mth root of a function-two function ratio. Appl. Math. Comput., 315:564-590, 2017. https://doi.org/10.1016/j.amc.2017.08.005.

[14] Á.A. Magreñán and I. Argyros. A contemporary study of iterative methods: convergence, dynamics and applications. Academic Press, 2018.

[15] J. Milnor. Dynamics in one complex variable. Annals of Mathematics Studies, 160. Princeton University Press, 2006.

[16] A.M. Ostrowski. Solutions of equations and systems of equations. Academic Press, New York-London, 1966.

[17] J.F. Traub. Iterative methods for the solution of equations. Prentice-Hall, Englewood Cliffs, 1964. 